\title{
HOW TO GET INFORMATION ABOUT TIPSTER
}

\author{
TIPSTER SE/CM \\ tipster@tipster.org
}

\section{HOW TO GET INVOLVED IN TIPSTER}

Determine if TIPSTER technology and the TIPSTER Architecture are what you need.

Contact : Government Representatives Relevant Documents: TIPSTER Concept Document TIPSTER Architecture Design

Gather requirements for your application and develop a Concept of Operation.

Contact: Government Representatives Documents to Read:

TIPSTER Concept Document TIPSTER Requirements Document TIPSTER CM Plan

Investigate what applications, similar to yours, have been implemented and by which contractors.

Contact : Government Representatives

Documents to Read: TIPSTER Concept Document TIPSTER ERB Reports Various TACAD's

Review previous designs involving TIPSTER technology, to support you design process.

Contact : Government Representatives

Documents to Read:

TIPSTER Concept Document

TIPSTER ERB Reports

Various TACAD's

Determine if your application can benefit from upgrading to advanced TIPSTER technology that has been developed since your application was implemented.

Contact : Government Representatives

Documents to Read:

TIPSTER Architecture Design

\section{POINTS OF CONTACT}

To FTP or Request Documents:

http://www.tipster.org

Government Representatives:

Dave Gunning

dgunning@arpa.mil

703-696-2218

Sarah Taylor sarah@ucia.gov

703-351-2565

John Prange

jdprang@afterlife.ncsc.mil

301-688-9739

George Thompson afthoga@dia.osis.gov

202-231-8370

Dick Barnett

k4guj@aol.com

703-696-1303

TIPSTER SETA:

Wiley Harris wileyv@aol.com 703-351-2572

TIPSTER SE/CM: James Conley tipster@tipster.org 703-556-1455

ARPA SETA:

Sharon Kaufmann skaufmann@snap.org 703-522-6067 